\title{
Characterization of Large, Heterogeneous Proteins by Electrospray Ionization-Mass Spectrometry
}

\author{
Lee Q. Huang and Anthony Paiva \\ Ramadas Bhat and Mel Wong \\ Biological Division, Bayer Corporation, Berkeley, California, USA
}

Bayer Research Center, Pharmaceutical Division, Bayer Corporation, West Haven, Connecticut, USA

\begin{abstract}
Characterization of heterogeneous proteins as large as 150,000 u was performed by a quadrupole mass spectrometer by using electrospray ionization (ESI). We were able to determine not only the molecular weight, but the detailed heterogeneity for the large glycoproteins as well. The successful application was facilitated by the optimization of the instrument in the high mass-to-charge range up to $m / z 4000$, where the multiply charged envelopes of the 150,000-u glycoproteins were found. For the analysis of clinically important monoclonal antibodies mass spectral data acquired by this method were consistent with the carbohydrate analysis and were useful in resolving the monosaccharide data into glycoform variations. In the case of the characterization of other large, heterogeneous proteins such as elongation factor 3 and bovine serum albumin, the quadrupole ESI mass spectrometer provided adequate mass resolution and high mass measurement accuracy to discern the modification and degradation of the proteins. (c) 1996 American Society for Mass Spectrometry (J Am Soc Mass Spectrom 1996, 7, 1219-1226)
\end{abstract}

$\mathrm{T}$ The revolution in molecular biology has been of enormous benefit to pharmaceutical research. Bioengineering is used routinely to produce proteins not only for direct therapeutic use, but also for facilitating the discovery of small molecules. The rapid development of biotechnology has, therefore, resulted in increasing demands for fast and accurate assays to characterize the proteins that are produced. Several questions crucial to protein research include the following: (1) Does the protein have the same amino acid sequence as indicated by the gene sequence? (2) Has the protein been modified post-translationally? (3) How pure is the protein after expression and purification? (4) Does the protein undergo degradation under different cellular or laboratory conditions? (5) How reproducible is the expression process from various cell lines or batches? Many of these questions can now be addressed rapidly and accurately by mass spectrometry.

Although mass spectrometry has traditionally been applied to the study of low molecular weight compounds, it recently joined the revolution in the biological sciences. This transition is mainly due to the development of electrospray ionization (ESI) and matrix-assisted laser desorption (MALD) mass spectrometry that enables intact protein molecules to be ionized

Address reprint requests to Dr. Lee Q. Huang, Bayer Research Center, Bayer Co., 400 Morgan Lane, West Haven, CT 06516. and accurately measured by a mass spectrometer [1, 2]. The sensitivity of these mass spectrometric techniques is in the low picomole range, similar to that of conventional bioassays such as sodium dodecylsulfate-polyacrylamide gel electrophoresis and amino acid analysis. However, mass spectrometry provides inherently superior accuracy and resolution when compared to these bioassays. As such, mass spectrometry, in conjunction with other bioanalytical methodologies, is rapidly becoming a standard technique for the analysis of biological samples.

After its invention [3], ESI found little use in mass spectrometry until Fenn and co-workers [2, 4, 6] demonstrated that this novel means could deliver multiply charged proteins into the gas phase for mass analysis. For many denatured proteins, the average charge state is approximately one positive charge per $1000 \mathrm{u}$. Today, molecules with molecular weight (MW) $<60,000$ can be analyzed easily by electrospray ionization-mass spectrometry (ESI/MS) [1]. For large, heterogeneous proteins, particularly glycoproteins, even the most current mass spectrometric instrumentation is still challenged because of the high demands in massto-charge range, mass resolution, and sensitivity.

Mass spectrometry analysis of intact monoclonal antibodies (MAbs) with MW of $150 \mathrm{ku}$ illustrates some of the difficulties. For example, a mass-to-charge range up to $\mathrm{m} / \mathrm{z} 4000$ is necessary to reveal the full pattern of ions generated by ESI because the protein is large 
and carbohydrates carry fewer charges. Moreover, high mass resolution is required for resolving glycoproteins ( $\sim 150,000 \mathrm{u}$ ) differing by one monosaccharide residue. These requirements pose extreme difficulty for a quadrupole mass spectrometer. ESI was employed in earlier studies to characterize intact MAbs by quadrupole [9-11], time-of-flight [12], and magnetic sector mass spectrometry [10]. Because of the lack of adequate high mass capability and/or mass resolution in these studies, ESI/MS was limited in the direct analysis of these large glycoproteins [9-12]. Although the molecular weights of the intact MAbs were measured by ESI/MS, it was not possible to determine the detailed microheterogeneities of the proteins. Therefore, microheterogeneities were only characterized when the protein was reduced to MAb fragments [11, 13, 14]. The present study demonstrates for the first time that ESI on a quadrupole instrument can provide information regarding not only the molecular weight for large glycoproteins $(150,000 \mathrm{u}$ ), but microheterogeneities as well. The applications of this method are illustrated in examples encountered in the research and development of large glycoproteins of pharmaceutical interest. The analysis of other large, heterogeneous proteins and mass measurement accuracy are also discussed.

\section{Experimental}

\section{Materials and Methods}

Bovine serum albumin (BSA), myoglobin, and L-methionyl-arginyl-phenylalanyl-alanine acetate $\cdot \mathrm{H}_{2} \mathrm{O}$ (MRFA) were obtained from Sigma Chemical Co. (St. Louis, MO) and were used without further purification. Human antitrypsin antibody was purchased from Calbiochem (San Diego, CA). Elongation factor 3 (EF-3) was obtained from Dr. K. Chakraburtty, University of Wisconsin at Green Bay. Proteins of MCP, ERK, and Raf were provided by Bayer Research Center (BRC, West Haven, CT). The peptide for tuning the instrument in the high mass range was synthesized in the core facility of BRC with a MW of 3259.6. The sequence of the peptide is AC QVIRNALASP GSGGDLGEAI REQPEQARLA L. The purification of MAbA and MAbB1,2,3 was performed in the following order: cation exchange chromatography on S-Sepharose (Pharmacia, Uppsala, Sweden), anion exchange chromatography on Q-Sepharose, and size exclusion chromatography on Sephacryl S-200HR. Solvents used were all high-performance liquid chromatography grade (J. T. Baker, Phillipsburg, NJ) and were used without further purification. All chromatography supports used in the purification of MAbs were purchased from Pharmacia LKB (Piscataway, NJ). Nitrogen gas was obtained from the boil-off of a 160-L pressure-building liquid nitrogen Dewar (Minnesota Valley Engineering, New Prague, MN) that provided dry, pure nitrogen at $200 \mathrm{lb} /$ in. $^{2}$.
A myoglobin and MRFA mixture was used for tuning and routine calibration of the instrument. The mixture was made in the following manner: stock solutions of myoglobin and MRFA were prepared by dissolving $0.9 \mathrm{mg}$ of horse skeletal muscle myoglobin in $1 \mathrm{~mL}$ of a solution of 50:50 methanol:water containing $5 \%$ acetic acid, and $0.6 \mathrm{mg}$ of MRFA was dissolved in $1 \mathrm{~mL}$ of a solution of 50:50 methanol:water containing $5 \%$ acetic acid. The calibration and tuning standard was made from the two stock solutions: $50 \mu \mathrm{L}$ of myoglobin and $12 \mu \mathrm{L}$ of MRFA were diluted with 538 $\mu \mathrm{L}$ of a 50:50 methanol:water solution in a polypropylene vial. Aliquots were stored at $-80^{\circ} \mathrm{C}$ and removed prior to use to minimize freeze/thaw effects. The tuning mixture was infused into the mass spectrometer at a rate of $3 \mu \mathrm{L} / \mathrm{min}$ on a Harvard Apparatus (model 22, South Natick, MA) syringe pump.

Monosaccharide composition analysis was performed on the desalted monoclonal antibodies by using the methanolysis procedure. Briefly, the samples were methanolyzed with $1-\mathrm{M}$ methanolic- $\mathrm{HCI}$ at $80^{\circ} \mathrm{C}$ for $24 \mathrm{~h}$. The sugar methyl glycosides were $\mathrm{N}$ acetylated in the presence of pyridine and acetic anhydride at room temperature followed by trimethylsilylation by using TRISIL reagent (Pierce, Rockford, IL). The separation and quantitation of various monosaccharides were achieved on a capillary gas chromatograph equipped with FID by using a 30-m DB-1 column (J \& W Scientific, Folsom, CA).

\section{Apparatus}

High-performance liquid chromatography was carried out by using a Michrom Bioresources (Auburn, CA) ultrafast microprotein analyzer (UMA) equipped with a 10-port automated sample injector and a variable wavelength $\mathrm{UV} /$ vis detector set at $280 \mathrm{~nm}$ with a $0.3-\mu \mathrm{L}$ (2-mm) flow cell. The microbore column was preceded by a microguard column (Michrom Bioresources). The eluents were A: $2 \%$ acetonitrile in water with $2 \%$ acetic acid and B: $98 \%$ acetonitrile in water with $2 \%$ acetic acid. Gradient elution from 0 to $100 \%$ B over $14 \mathrm{~min}$ at a flow of $100 \mu \mathrm{L} / \mathrm{min}$ on a PLRP-S (Michrom Bioresources) $8-\mu \mathrm{m} 1000-\AA 1.0-\mathrm{mm} \times 50$ $\mathrm{mm}$ microbore column provided separation of the proteins from their accompanying salts in less than 15 $\min$.

Electrospray ionization was performed on a Finnigan TSQ 7000 triple quadrupole mass spectrometer (Finnigan MAT; San Jose, CA) fitted with a Finnigan ESI interface. Protein samples were introduced without any splitting into the electrospray interface through an untreated fused-silica capillary with a $50-\mu \mathrm{m}$ i.d. and $190-\mu \mathrm{m}$ o.d. coupled to the high-performance liquid chromatograph (HPLC) with a stainless steel zero dead-volume union (Upchurch Scientific, Oak Harbor, WA). A positive voltage of $4500-5000 \mathrm{~V}$ was maintained on the electrospray needle throughout all the 
experiments. The ESI capillary was heated to $200{ }^{\circ} \mathrm{C}$. Nitrogen was introduced as a sheath gas at a pressure of $50 \mathrm{lb} /$ in. $^{2}$ and auxiliary nitrogen gas at $30 \mathrm{~mL} / \mathrm{min}$ was also introduced to improve sensitivity and reproducibility.

Quadrupole 1 was operated in the high mass-tocharge range and was scanned from $\mathrm{m} / z 1800$ to 4000 over $5 \mathrm{~s}$ for the MAbs, EF-3, and BSA. The electron multiplier was set at $1200-1400 \mathrm{~V}$. This setting resulted in a typical total ion count (TIC) of $1 \times 10^{8}$ counts. All protein spectra were acquired in profile mode. Profile data were averaged by using a peak width of $m / z 100 \mathrm{mu}$ for the large proteins. A mass defect of $m / z 0 \mathrm{mu}$ at $\mathrm{m} / \mathrm{z} 0$ and $\mathrm{m} / \mathrm{z} 300 \mathrm{mu}$ at $\mathrm{m} / \mathrm{z}$ 1000 was used for data processing. Mass deconvolution of multiply charged ions was performed by using Finnigan biochemistry software.

\section{Results and Discussion}

\section{Mass Spectrometry Tuning and Mass Spectrum for Antitrypsin Antibody}

Our preliminary data and previous studies [9-11] indicated that the ion envelopes for MAbs were observed in the high mass-to-charge range $(\mathrm{m} / \mathrm{z}$ 2000-4000) during electrospray ionization. Therefore, we used a synthetic peptide that gives a singly charged ion at $\mathrm{m} / z 3260.6$ to tune and determine the mass resolution at the high mass-to-charge range. Peak profiles (see Figure 1) were examined to determine the proper parameters for mass resolution. Figure 1c (showing a peak width at half height of $m / z \sim 6$ ) illustrates a typical tuning result in the high mass-to-charge range, where lower resolution is tuned to maintain sensitivity. Figure $1 \mathrm{~b}$ shows the resulting peak profile when the mass spectrometer was tuned at a slightly higher resolution (approximately $m / z$ 3-u peak width at half the height of the peak). Figure 1a illustrates the highest resolution achieved in which unit mass resolution was demonstrated in this high mass-to-charge range; the isotope peaks at the $\mathrm{m} / \mathrm{z} 3260.6$ were clearly resolved. The sensitivity of the instrument at these three resolution settings was measured by the number of ion counts detected as marked in the upper right corner of the Figures $1 \mathrm{a}-\mathrm{c}$. The peak heights were 552,340, 186,679 , and 11,757 counts for low (Figure 1c), medium (Figure 1b), and high resolution (Figure 1a), respectively. It is apparent that the sensitivity decreased only threefold from low to medium resolution, but dropped dramatically, that is, almost 50 -fold, when the instrument was tuned at unit mass resolution at the high mass-to-charge range.

One type of glycosylation heterogeneity of an MAb may arise from the incomplete substitution or sequential deletion of monosaccharide residues in the sugar chain, which would result in several protein components differing by the residue masses, for example, galactose $(162 \mathrm{u})$, fucose $(146 \mathrm{u})$, or sialic acid $(291 \mathrm{u})$.
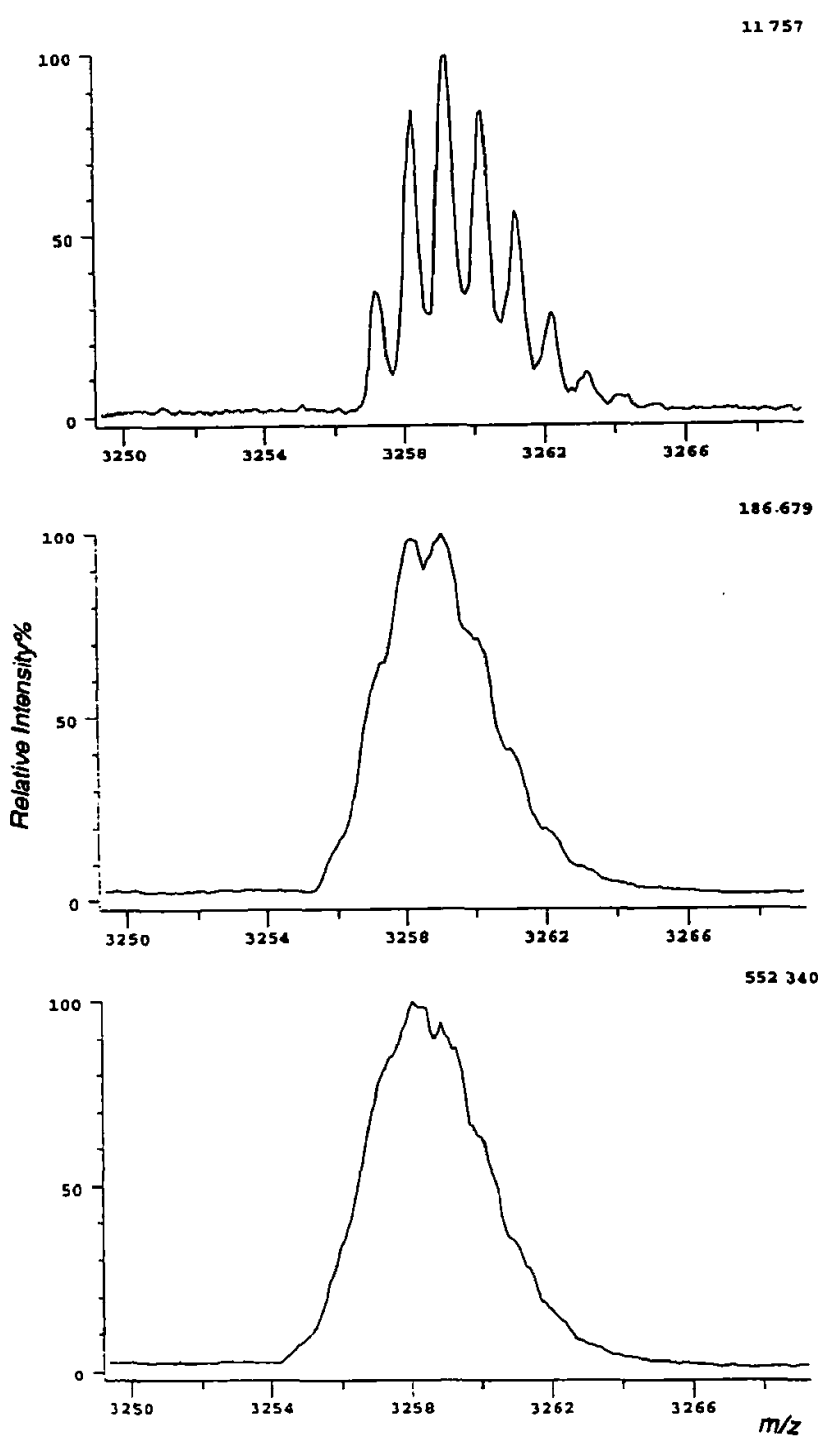

Figure 1. Peak profiles of a peptide with a molecular weight of 3259.6 when tuned in high (top), medium (middle), and low (bottom) mass resolution.

To detect this type of microheterogeneity of MAbs, it is necessary to achieve a mass resolution that resolves components differing by $\sim 150 \mathrm{u}$ (the average residue mass of a monosaccharide) in the mass range of 150,000 $\mathrm{u}$ (the average MW of MAbs) after the deconvolution of the multiply charged envelopes. Since we observed in preliminary experiments that the MAbs carried $\sim 50$ charges, the actual instrumental mass resolution required was $m / z \sim 3 \mathrm{u}$ (150 u/50 charges). The objective was to tune the mass spectrometer for adequate mass resolution to detect different glycosylated forms of the proteins without significant loss of sensitivity; thus, medium resolution $(\mathrm{m} / \mathrm{z} 3 \mathrm{u})$ in the mass-tocharge range of $\sim 3000$ was chosen for this study.

The ESI mass spectrum of the antitrypsin antibody, a $\mathrm{MAb}$, was acquired by using the aforementioned conditions; the spectrum is shown in Figure 2. Antitrypsin antibody is a commercially available glycoprotein and has been characterized biologically [15] as 


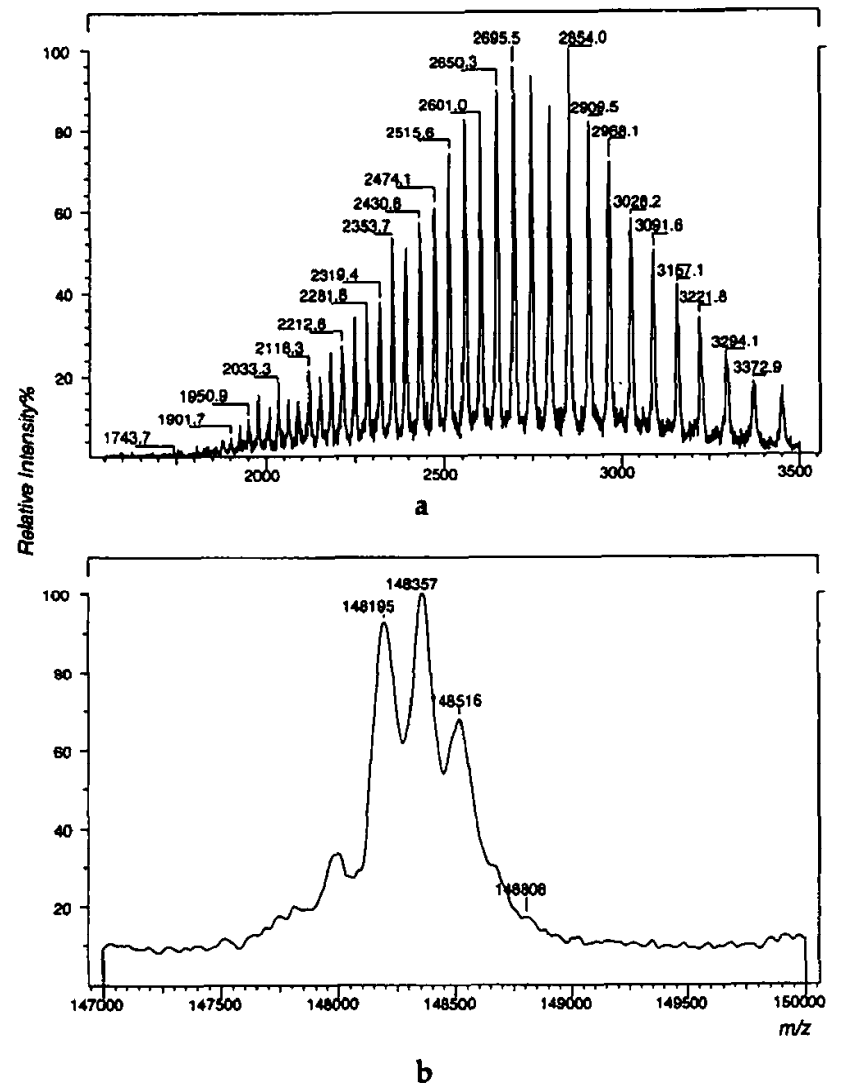

Figure 2. A mass-to-charge ratio spectrum (a) and deconvoluted mass spectrum (b) of the antitrypsin antibody.

well as by mass spectrometry [9]. The upper panel of Figure 2 shows the multiply charged ion envelope for the antibody, which was then subjected to a deconvolution algorithm to generate the final spectrum. As predicted, a resolution of $m / z$ 3-u resolved multiple peaks in each multiply charged state because of the heterogeneity of the glycoprotein. After the deconvolution of the acquired data, the peaks corresponding to individual hexose sugar deletions $(\sim 162 \mathrm{u})$ were clearly identified as shown in the lower panel of Figure 2 . The masses of the constituents $(148,195 ; 148,357$; and $148,516 \mathrm{u})$ were in agreement with the early measurement of the average mass of $148,484 \mathrm{u}$ for the same source of the protein [9]; however, the heterogeneity of the protein had not been resolved in the previous study [9]. Our results suggest that it is possible for a quadrupole mass spectrometer to measure the $150-\mathrm{ku}$ glycoproteins and resolve the individual glycosylation heterogeneity.

\section{Characterization of Other Monoclonal Antibodies}

Four other MAbs of clinical significance-MAbA, MAbB1, MAbB2, and MAbB3 - were also analyzed in our study, and the results are discussed in subsequent text. The spectrum of MAbA is given in Figure 3. The upper panel illustrates the multiply charged ions; the peaks that were due to microheterogeneities for every
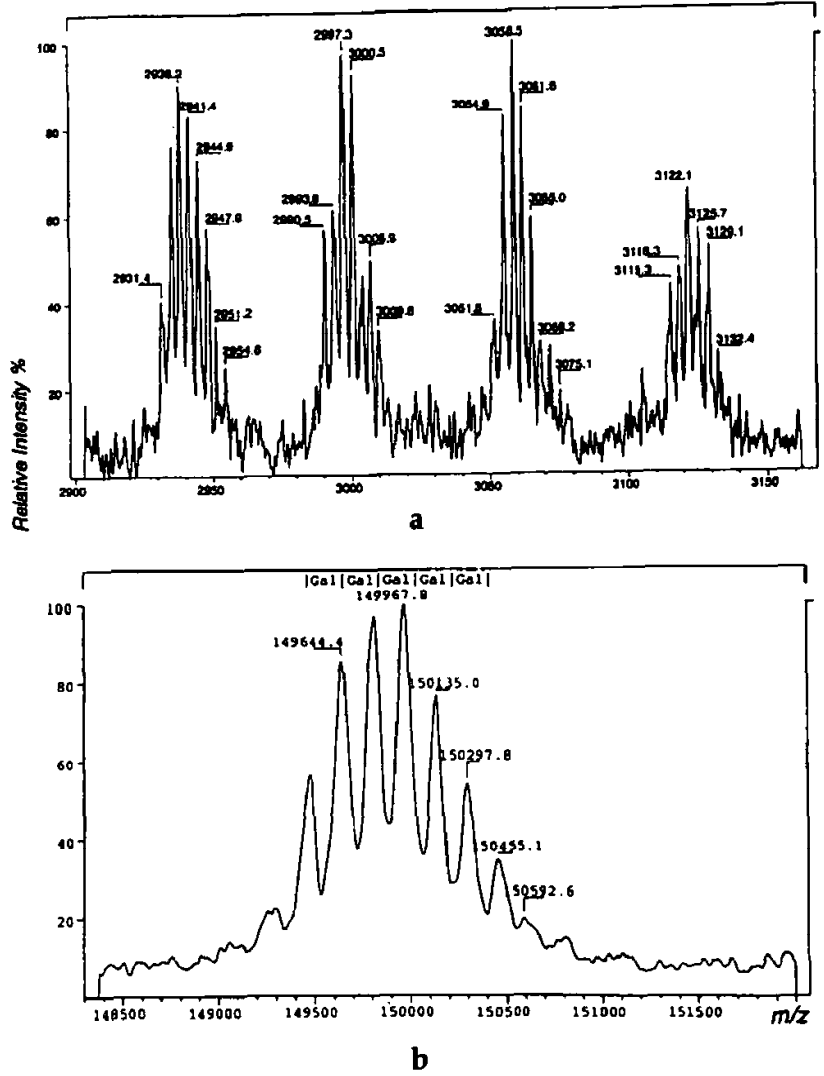

Figure 3. Mass-to-charge ratio spectrum (a) and deconvoluted mass spectrum (b) of the MAbA antibody.

charge state were well resolved. The deconvoluted spectrum is shown in the lower panel of Figure 3; here, it is evident that at least seven constituents were detected in this sample because of the heterogeneity. The mass difference of $\sim 162 \mathrm{u}$ among the constituents suggests that the heterogeneity is due to hexose deletion. Carbohydrate analysis has shown that the MAb has $\alpha$-galactose residues that are partially substituted on the terminal $\beta$-galactose residues (data not shown here). Therefore, the seven constituents that mass spectrometry detected are likely due to the sequential deletion of the galactose residues on the complex $\mathrm{N}$-linked chains.

From the deconvoluted electrospray spectra of MAbB1, MAbB2, and MAbB3 in Figure 4, the following points were observed: (1) the measured $M W$ of the MAbB1 was considerably higher (at least $1500 \mathrm{u}$ higher) than those of MAbB2 and MAbB3; (2) although the measured MWs of the MAbB2 and MAbB3 were similar $(\sim 148,486 \mathrm{u})$, the extent of glycosylation heterogeneity of the proteins from the MAbB3 was much higher than that of the MAbB2; $(3)$ the observed heterogeneity is similar for the MAbB1 and MAbB2 even though the measured MWs of the two proteins were different.

Data from total carbohydrate composition analysis for the antibodies were used to interpret the observations (Figure 5). Overall, the MAbB1 antibody con- 

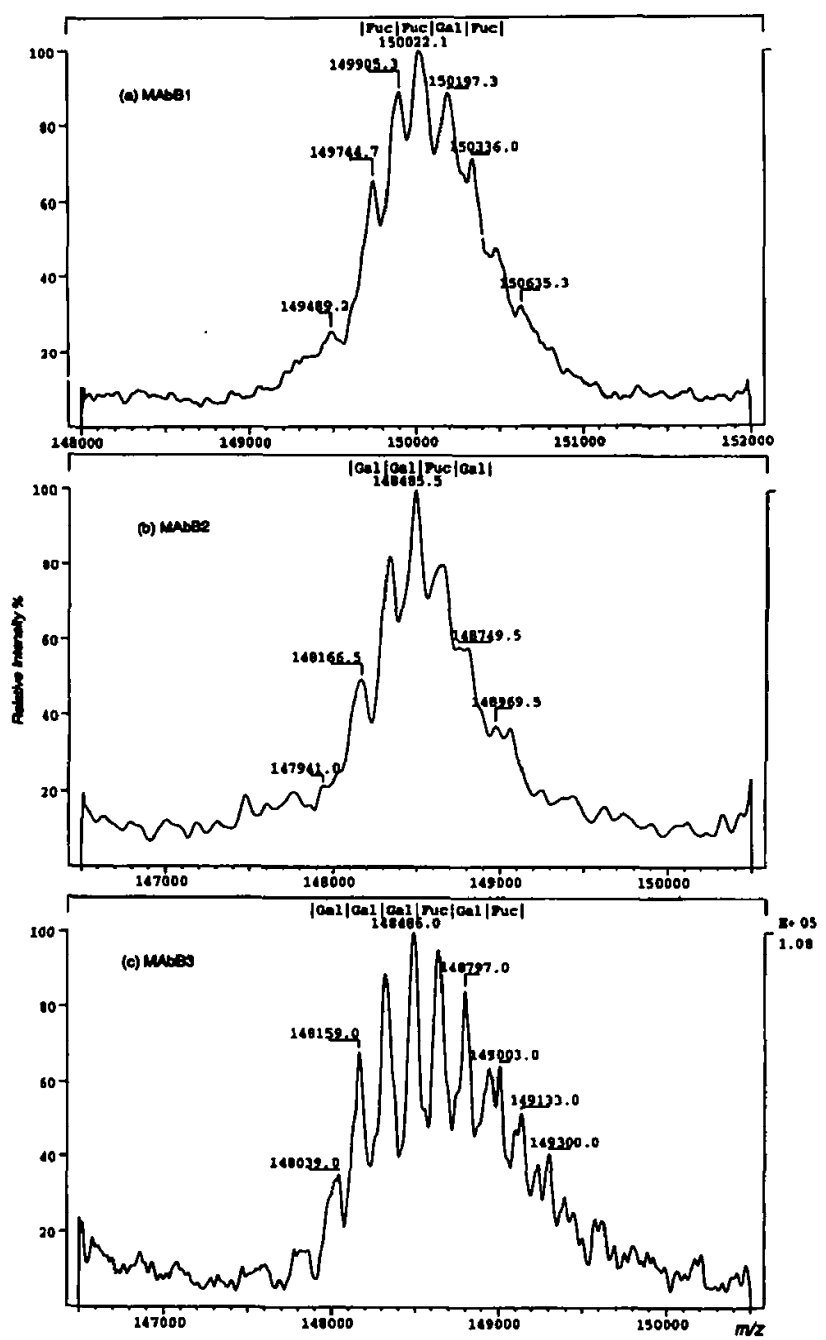

Figure 4. Deconvoluted mass spectra of (a) MAbB1, (b) MAbB2, and (c) MAbB3.

sisted of higher glycosylation levels, that is, 150 $\mathrm{mol} / \mathrm{mg}$ IgG, when compared to the MAbB2 (115 $\mathrm{nmol} / \mathrm{mg} \mathrm{IgG)}$ and MAbB3 (112 nmol/mg IgG). Higher levels of total carbohydrates in MAbB1 suggest that the higher $(\sim 1500 \mathrm{u}) \mathrm{MW}$ detected by mass spectrometry for the MAbB1 is attributable to the car-

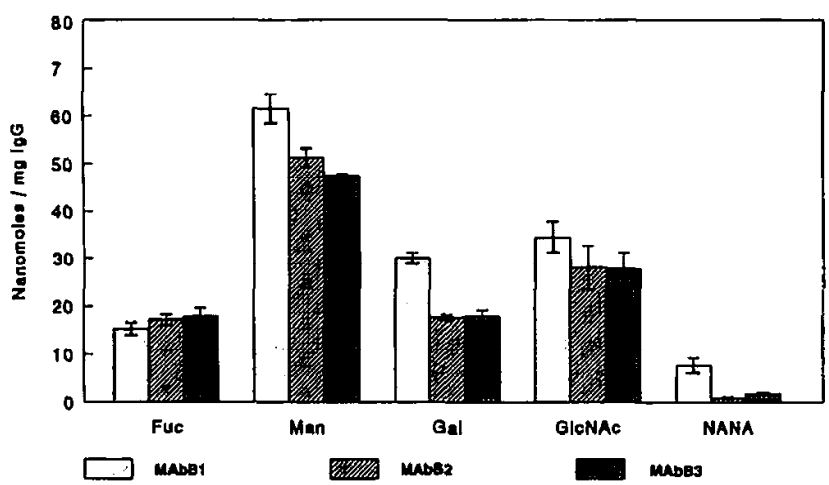

Figure 5. Monosaccharide compositions of MAbB1, MAbB2, and MAbB3. bohydrate composition. Typically, all IgG molecules incorporate two $\mathrm{N}$-linked oligosaccharide chains each on the Asn-297 of the $\mathrm{CH}_{2}$ heavy chain domain. In addition, glycosylation of the variable regions of heavy or light chains in the Fab arm has also been reported. Both mass spectrometry detection and carbohydrate analysis data in the present study indicate that MAbB1 may consist of more than two glycan chains, that is, glycosylation in the Fab region. This additional oligosaccharide chain results in the macroheterogeneity among MAbB1, MAbB2, and MAbB3.

The multiple mass peaks detected by mass spectrometry for each of the antibodies of MAbB1, MAbB2, and MAbB3 were due to the occurrence of extremely extensive microheterogeneity. The microheterogeneity detected by mass spectrometry revealed that the multiple constituents differed either by $\sim 162$ or $\sim 146 \mathrm{u}$ for all the antibodies. Carbohydrate analysis showed that galactose (Gal; 162 u) or fucose (Fuc; $146 u$ ) residues were substituted on the terminal $N$-linked chains; thus, the microheterogeneity of these MAbs was thought to be caused by the sequential deletion of Gal or Fuc from the terminus.

More specifically, mass spectrometry data showed that MAbB1 had more deletions of Fuc than those of MAbB2 and MAbB3. This finding correlated well with the monosaccharide composition results: MAbB1 had a mannose to fucose molar ratio of 3.0:0.7 based on the three mannose residues per $\mathrm{N}$-glycan chain as compared with a molar ratio of 3.0:0.95 for MAbB2 and MAbB3. Therefore, both mass spectrometry and monosaccharide composition data suggested that fucosylation of the $N$-linked oligosaccharides was incomplete for the MAbB1. With regard to the degree of galactosylation, a maximum of two galactose (Gal) residues could be present on the antibodies for every three mannose (Man) residues per glycan chain (complex, biantennary type), that is, a homogeneous glycoprotein without $\mathrm{Gal}$ deletion would give a ratio of 3.0:2.0. However, the mannose to galactose molar ratios determined by carbohydrate analysis were $3.0: 1.5$, 3.0:1.0, and 3.0:1.1 for MAbB1, MAbB2, and MAbB3, respectively. This carbohydrate structural variation can now be interpreted by using the mass spectrometry data. The lower than expected ratio is due to the deletion of galactose residues as detected by mass spectrometry. Also, more galactose deletions in MAbB2 and MAbB3 than in MAbB1 as indicated in Figure 4 may account for even lower Man:Gal ratios for MAbB2 and MAbB3. Thus, mass spectrometry data were very useful in resolving the monosaccharide analysis data into glycoform variations, arising from the galactosylation of $\mathrm{N}$-linked glycans.

It was interesting to note in our study by using $2 \%$ acetic acid in the ESI solvent (Finnigan ESI quadrupole mass spectrometer) that the charge-state distributions for all the MAbs were in the mass-to-charge range of 2000-3500 u/e, a much higher range than those observed for other proteins $(800-2000 \mathrm{u} / \mathrm{e})$. This high 
mass-to-charge ratio distribution was also observed in other studies for MAbs at different conditions such as $1 \%$ formic acid (Fisons/VG ESI quadrupole mass spectrometer) $[10,11]$ and $4 \%$ acetic acid (homemade ESI/TOF-MS) [12]. The large size of the MAbs may not be the sole cause for such a high mass distribution. Typical 3-5\% glycosylation of the MAbs may account, in part, for the shift of the envelope to high mass-tocharge range.

Although the optimization of the mass spectrometer in high mass-to-charge range is crucial to the characterization of MAbs, we observed that it is also important to introduce large proteins into the electrospray through a HPLC. This process eliminated the formation of $\mathrm{Na}$ and $\mathrm{K}$ adduct ions, and thus improved the "apparent resolution." In fact, we found that the multiply charged ion envelope for large proteins was seriously congested due to adduct ion formation even when the samples were carefully dialyzed against water prior to infusion into the mass spectrometer.

\section{Analysis of Elongation Factor 3 and Bovine Serum Albumin}

Although mass spectrometry characterization of MAbs represents a challenging case to current mass spectrometry instrumentation, it is also important to have high mass-to-charge range and relatively high resolution of mass spectrometry for the characterization of other large or heterogeneous proteins. Figure 6 illustrates the mass spectrum for a large protein-elongation factor 3 (EF-3) -with a MW of $116 \mathrm{ku}$. EF-3 is an important protein involved in protein transcription and biosynthesis in yeast. While proteins with MW $<60$ $\mathrm{ku}$ are becoming more routine, proteins like EF-3 with MW $>100 \mathrm{ku}$ still present difficulties for ESI/MS. In our preliminary experiment we encountered difficulty in the analysis because of the congestion in the multiply charged ion envelope of the mass spectrum, particularly in high charge states, for example, $>100$ charges. When relatively high mass resolution was tuned and the sample was introduced through a HPLC column, the spectrum in Figure 6 was achieved, with a multiply charged ion envelope that was well resolved. This protein generated over 100 multiply charged peaks with charge states from 40 to 155 , not commonly observed for other proteins.

Bovine serum albumin (BSA) is a standard protein in biological research, and it has been characterized by several mass spectrometry laboratories [16-18]. The measured molecular weights of BSA range from 65,000 to $67,000 \mathrm{u}$ in ESI experiments performed in both quadrupole and Fourier transformation-ion cyclotron resonance instruments. In recent studies, Feng et al. [19] reported the measured BSA MW of $66431.5 \mathrm{u}$, which is consistent with the MW of 66,430.3 u suggested by an independent sequence study [18]. BSA from the same source (Sigma) was subject to ESI/MS analysis in our study, and the spectrum is given in

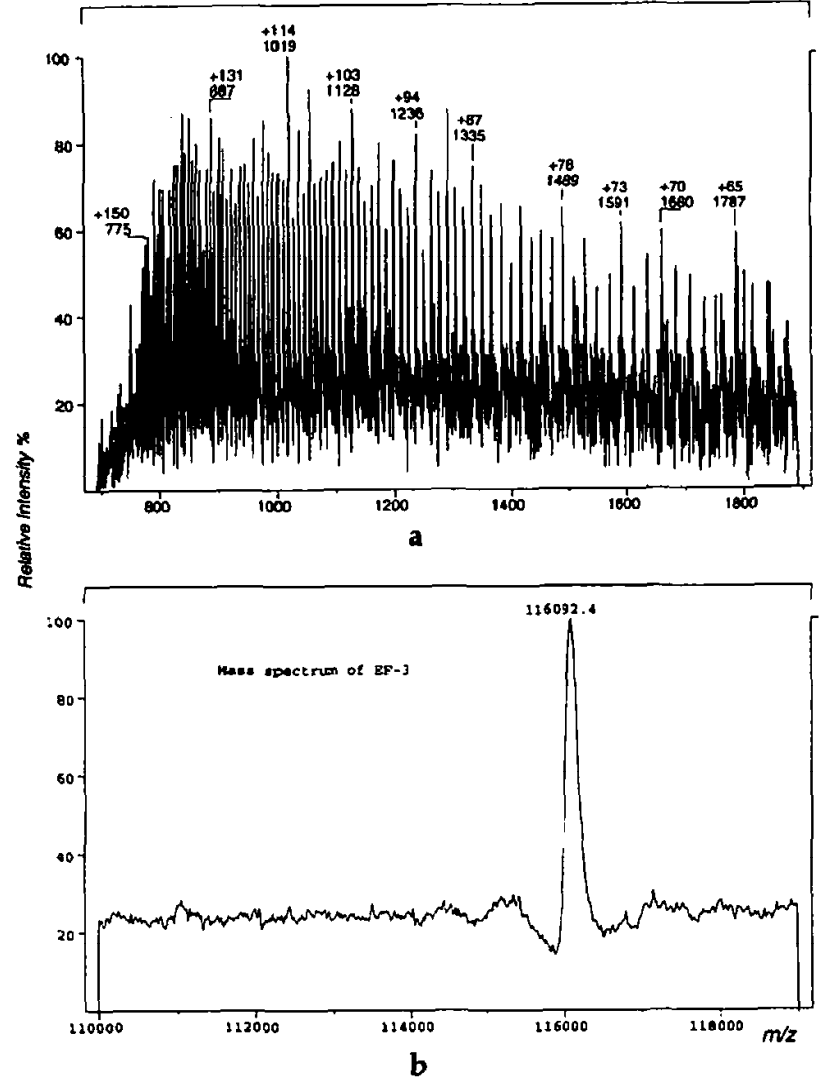

Figure 6. Mass-to-charge ratio spectrum (a) and deconvoluted mass spectrum (b) of the EF-3.

Figure 7. The spectrum shows that the protein was heterogeneous and that the major component had a MW of $66,430.1 \mathrm{u}$, which agreed with the predicted MW of $66,430.3$ by gene sequence [18]. The origin of the multiple peaks still awaits further characterization. It is now clear that the inconsistency that resulted from many previous mass spectrometry measurements occurred not because of the poor accuracy of ESI/MS, but because of the inherent heterogeneity of the BSA. The reported [16-18] MWs of BSA were actually the average MWs of the multiple components as there was limited mass resolution in the analyses.

If the heterogeneity can, in fact, be resolved, there would be a significant improvement in the mass measurement accuracy by ESI for large proteins. It is generally believed that the best mass measurement accuracy of proteins is $0.01 \%$ by ESI/MS [1]. This indicates that the absolute error would be greater for large proteins, for example, \pm 6 for $60,000-u$ and \pm 10 for $100,000-\mathrm{u}$ proteins [13]. However, this assumption overlooks the fact that multiple measurements are performed by ESI/MS because of the multiply charged ion phenomena, that is, every charge state is used once to calculate the molecular weight of the protein. Since both the standard deviation and standard error are inversely proportional to the number of measurements (n) [20], the precision and accuracy for protein mass measurement are not simply limited by the quadrupole 


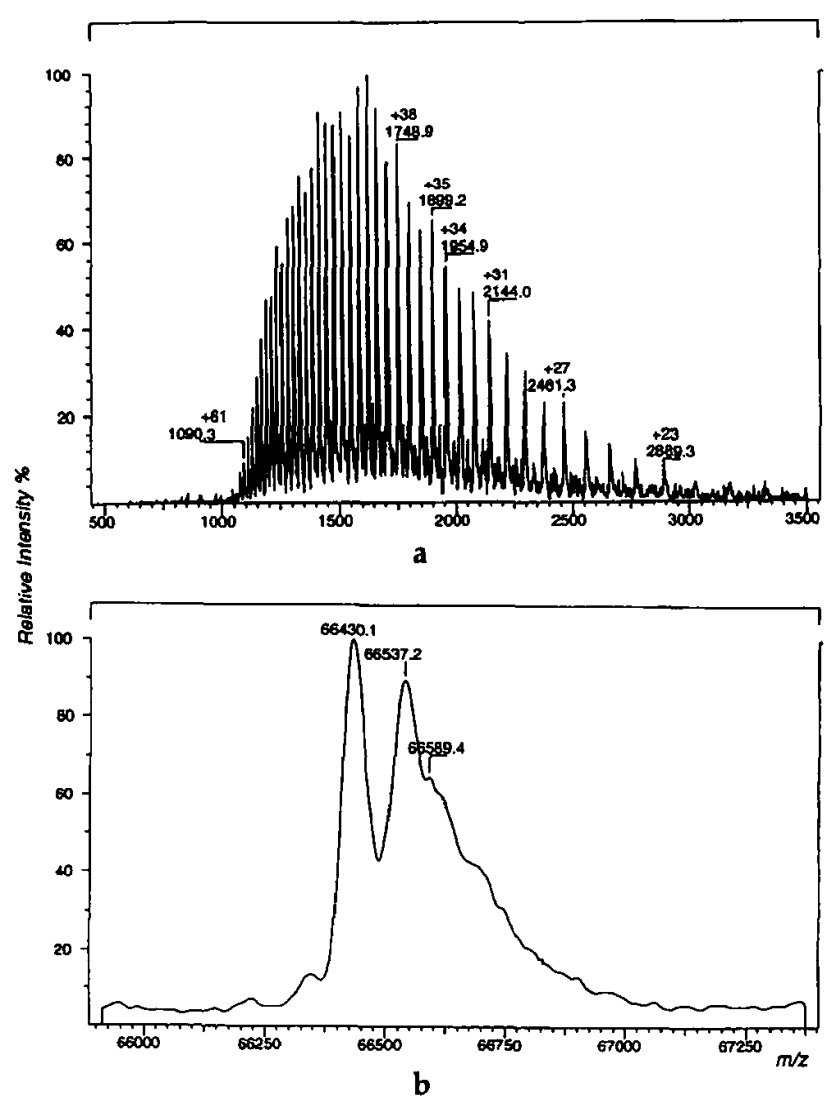

Figure 7. Mass-to-charge ratio spectrum (a) and deconvoluted mass spectrum (b) of the BSA.

precision and accuracy of a single peak. Furthermore, many large proteins generate more multiply charged peaks, that is, more measurements; therefore, this larger $n$ results in a smaller standard deviation and standard error. For example, almost 50 multiply charged peaks for BSA (66 ku; Figure 7) and 100 for EF-3 (116 ku; Figure 6) were generated for the MW measurement as compared to typically $\sim 10$ peaks for a 10-ku protein. Table 1 lists error analysis for various proteins with known theoretical gene sequences in our study. The relative errors were much smaller than $0.01 \%$. The mass measurement accuracy for large proteins such as MAbs cannot be assessed at this time

Table 1. Results of measured MWs by ESI/MS for various proteins

\begin{tabular}{lrrrc}
\hline Protein & $\begin{array}{c}\text { Theoretical } \\
\text { MW (Da) }\end{array}$ & $\begin{array}{c}\text { Measured } \\
\text { MW (Da) }\end{array}$ & $\begin{array}{c}\text { Error } \\
\text { (Da) }\end{array}$ & $\begin{array}{c}\text { Relative } \\
\text { error (\%) }\end{array}$ \\
\hline \hline MIP2 & 7845.35 & 7845.6 & -0.25 & 0.0032 \\
hMCP-1 & 8665.02 & 8665.0 & +0.02 & 0.0002 \\
RAF & 8946.45 & 8946.5 & -0.05 & 0.0006 \\
mMCP-1 & 9860.59 & 9860.0 & +0.59 & 0.0060 \\
IL-4 & 15088.43 & 15089.0 & +0.57 & 0.0038 \\
Stromelysin & 19605.92 & 19605.0 & +0.92 & 0.0047 \\
GST & 26984.60 & 26984.6 & +0.00 & - \\
ERK & 41144.58 & 41145.0 & -0.42 & 0.0010 \\
BSA & 66430.30 & 66430.1 & -0.20 & 0.0003 \\
\hline
\end{tabular}

because of the difficulty in obtaining exact MW for these proteins.

\section{Conclusions}

Magnetic sector instruments and Fourier transformmass spectrometry may potentially provide superior high mass resolution and mass-to-charge range for the analysis of complicated protein samples. However, an ESI quadrupole mass spectrometer is still the most widely used configuration in industrial research because of its simplicity and adaptability to ESI. Our study showed that a quadrupole mass spectrometer with electrospray ionization can obtain adequate mass resolution and mass-to-charge range to detect both the molecular weight and the microheterogeneity for $150,000-u$ glycoproteins. In our study, relatively high mass resolution allows differentiation of multiple components of heterogeneous proteins, thus improving MW measurement accuracy for proteins. We also observed that charge-state distribution of MAbs during electrospray ionization is in the $\mathrm{m} / z$ 2000-3500 range as compared to $m / z 800-2000$ for most proteins, which is consistent with earlier studies [10-12]. It should be emphasized that mass spectrometry data are complemented by information from other techniques such as carbohydrate analysis, Edman sequencing, and amino acid analysis in order to understand and solve biological problems.

\section{Acknowledgments}

We thank Dr. Mark Sanders of Finnigan MAT for technical assistance. We also thank J. Kupcho, C. Brini, and G. Davis for performing amino acid analysis and Edman sequencing. The authors are indebted to S. Roczniak, H. Roder, K. Chakraburtty, and many others not named here for providing quality protein samples in this study. Finally, R. Tilton's input and useful discussions on this research are gratefully acknowledged.

\section{References}

1. Chait, B. T.; Kent, S. B. H. Science 1992, 257, 1885-1894.

2. Fenn, J. B.; Mann, M.; Meng, C. K.; Wong, S. F.; Whitehouse, C. M. Science 1989, 246, 64-71.

3. Dole, M.; Mack, L. L.; Hines, R. L.; Mobley, R. C.; Ferguson, L. D.; Alice, M. B. J. Chem. Phys. 1968, 49, 2240-2249.

4. Fenn, J. B.; Mann, M.; Meng, C. K.; Wong, S. F.; Whitehouse, C. M. Mass Spectrom. Rev. 1990, 9, 37-70.

5. Carr, S. A.; Hemling, M. E.; Bean, M. F.; Roberts, G. D. Anal. Chem. 1991, 63, 2802-2824.

6. Wong, S. F.; Meng, C. K.; Fenn, J. B. I. Phys. Chem. 1988, 92, 546-550.

7. Covey, T. R.; Bonner, U.; Ingendoh, A.; Henion, J. D. Rapid Commun. Mass Spectrom. 1988, 2, 249-256.

8. Smith, R. D.; Loo, J. A.; Ogorzalek-Loo, R. R.; Busman, M.; Udseth, H. R. Mass Spectrom. Rev. 1991, 10, 359-452.

9. Feng, R.; Konishi, Y. Anal. Chem. 1992, 64, 2090-2095.

10. Bennett, K. L.; Hick, L. A.; Truscott, R. J. W.; Sheil, M. M. I. Mass Spectrom. 1995, 30, 769-771.

11. Ashton, D. S.; Beddell, C. R.; Cooper, D. J.; Craig, S. J.; Lines, 
A. C.; Oliver, R. W. A.; Smith, M. A. Anal. Chem. 1995, 67 835-842.

12. Verentchikov, A. N.; Ens, W.; Standing, K. G., Anal. Chem. 1994, 66, 126-133.

13. Bourell, J. H.; Clauser, K. P.; Kelley, R.; Carter, P.; Stults, J. T. Anal. Chem. 1994, 66, 2088-2095.

14. Lewis, D. A.; Guzzetta, A. W.; Hancock, W. S.; Costello, M. Anal. Chem. 1994, 66, 585-596.

15. Zegers, N. D.; Claassen, E.; Gerritse, K.; Deen, C.; Boersma, W. J. A. Clin. Chem. 1991, 37, 1606-1611.

16. Chowdhury, S. K.; Katta, V.; Chait, B. T. Proceedings of the 38th ASMS Conference on Mass Spectrometry and Allied Topics; Tucson, AZ, June 3-8, 1990; pp 138-139.

17. Henry, K. D.; Wang, B. H.; Williams, E. R. Proceedings of the 38th ASMS Conference on Mass Spectrometry and Allied Topics; Tucson, AZ, June 3-8, 1990; pp 433-434.

18. Hirayama, K.; Akashi, S.; Furuya, M.; Fukuhara, K. Biochem. Biophy. Res. Commun. 1991, 173, 639-646.

19. Feng, R.; Konishi, Y.; Bell, A. W. I. Am. Soc. Spectrom. 1991, 2, 387-401.

20. Fritz, J. S.; Schenk, G. H. Quantitative, Analytical Chemistry, 4th ed.; Allyn and Bacon: Boston, 1979; Chap 3. 